\title{
Anisocoria Secondary to Anticholinergic Mydriasis from Homeopathic Pink Eye Relief Drops
}

\author{
Lin Chen, MD, PhD; Joseph C. Yeung, MD; and Dennis R. Anderson, MD
}

\begin{abstract}
A woman, aged 70 years, developed anisocoria after applying homeopathic eye drops (Similasan Pink Eye Relief) to her left eye. Her pupil was dilated for two weeks and did not respond to light or near stimuli for one week. Both $0.1 \%$ and $1 \%$ pilocarpine failed to constrict her left pupil, and magnetic resonance imaging of her brain did not reveal any abnormality. The eye drops she had used contain belladonna extracts which have a natural atropine component. This case demonstrates the importance, when evaluating a patient presenting with anisocoria, of knowing the chemical ingredients of the homeopathic eye drops, which often are not listed.
\end{abstract}

Keywords: Anisocoria; Anticholinergic; Belladonna; Mydriasis; Homeopathic

$\Lambda$ nisocoria could suggest a life-threatening event due to its association with cranial third-nerve palsy or Horner's syndrome. Pharmacological mydriasis is one of the benign etiologies of anisiocoria. Although it is common knowledge that the vasoconstrictor in over-thecounter (OTC) eye drops can cause adrenergic mydriasis, ${ }^{1}$ there has never been a published report on the pupillary effect from OTC homeopathic eye drops.

Homeopathy, treating illness with a holistic and natural approach, has become a popular alternative medicine practice, even in the Western world. ${ }^{2}$ Homeopathic eyedrops (from companies such as Similasan, Highlands Ranch, Colorado), derived from natural sources are now readily available at local retail stores. Two of the nine OTC drops produced by Similasan contain active ingredients obtained from Atropa belladonna, a perennial herbaceous plant commonly known as deadly nightshade. The extracts of the plant have long been used to dilate the pupil in the treatment of iritis due to its tropane alkaloids (atropine, scopolamine, and hyoscyamine). ${ }^{3}$ This report describes a case of anisocoria secondary to anticholinergic mydriasis caused by use of Similasan's Pink Eye Relief drops.

\section{Case Report}

A Caucasian woman, aged 70 years, presented with a 5-day history of dilated left pupil. She denied any vision problems, eye pain, double vision, or eyelid drooping. She had no headache, dizziness, or imbalance. She reported that 5 days prior, when she noticed a larger pupil as well as some irritation in the left eye, she started self-treating her left eye twice a day with an OTC "pink eye relief" drop. She stopped using the drops 2 days prior to presentation because they were not effective. She denied using any other drops, perfume spray, or motion sickness patch. She had a medical history of hypertension, hyperlipidemia, and breast cancer, but no history of diabetes mellitus. She had previous annual eye examinations with documented equal pupil size.

On examination, her vision was $20 / 25$ for each eye. Her right pupil was $3 \mathrm{~mm}$ and $5 \mathrm{~mm}$ under bright and dim light, respectively; however, her left pupil remained at $7.5 \mathrm{~mm}$ with no constriction to light or near stimulation. There was no left relative afferent pupil defect by reverse, no left ptosis, nor extraocular movement abnormality. Intraocular pressure, anterior segment, and posterior segment were unremarkable, except for mild nucleus sclerosis bilaterally and left choroidal nevus. Upon investigation, it was found that the OTC 


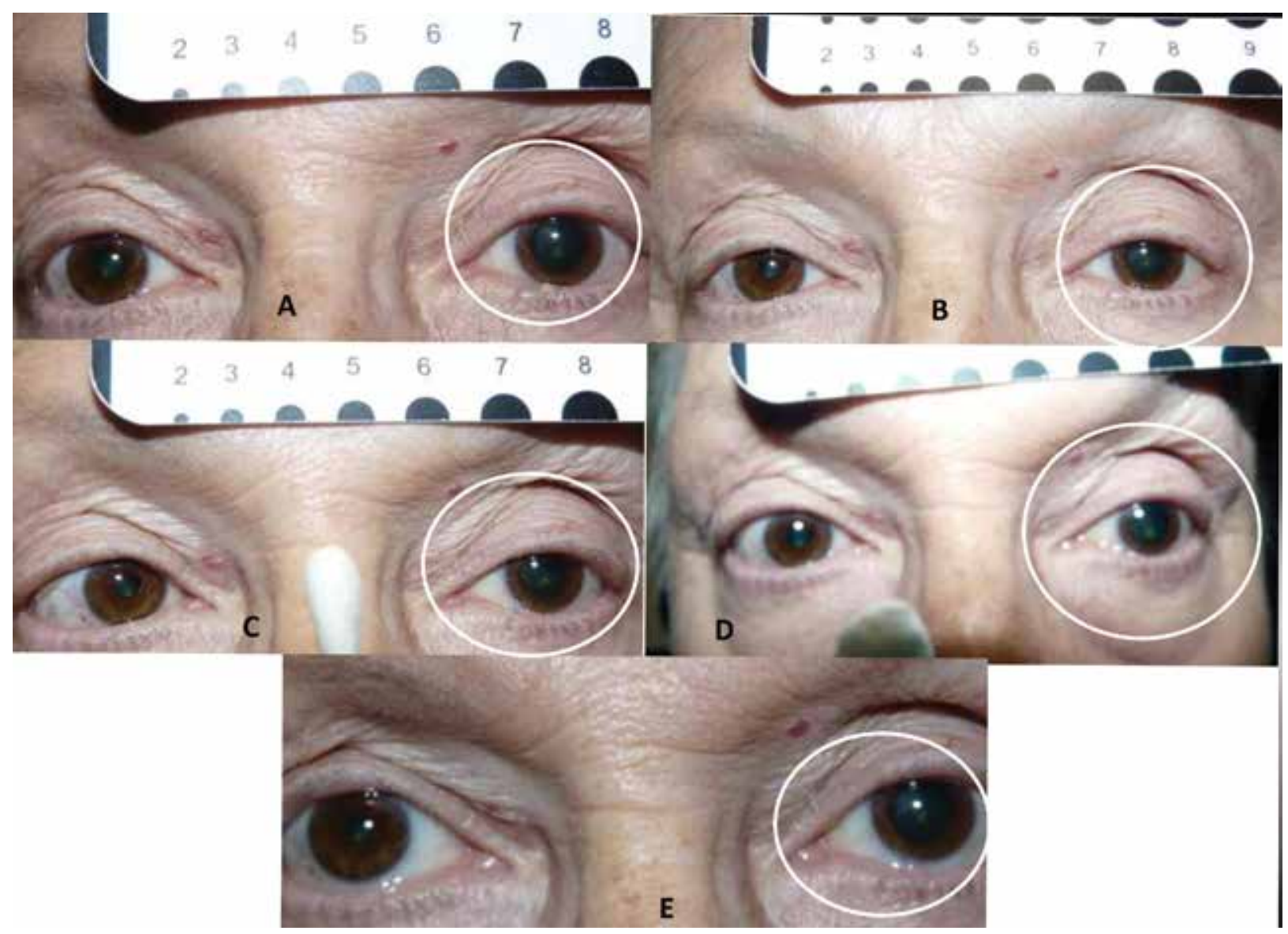

Figure 1. Pilocarping pupil test showing (A) the dilated left pupil which did not respond to (B) light, (C) near stimuli, (D) $0.1 \%$ pilocarpine, or (E) $1.0 \%$ pilocarpine.

Similasan Pink Eye Relief drops, which the patient had used, contained the following active ingredients: Belladonna $6 \mathrm{X}$, Euphrasia 6X, and Hepar sulphuris 12X.

The following day, which was the third day after the patient stopped her OTC drops, a $0.1 \%$ and $1 \%$ pilocarpine test was performed which demonstrated that her left pupil failed to constrict (Figure 1). Three days later (Day 6 off the drops), her left pupil, although still dilated to $7 \mathrm{~mm}$, began to respond slightly to light $(6.5 \mathrm{~mm})$ and near stimuli $(6 \mathrm{~mm})$. However, one week later, (Day 12 off the drops), her left pupil was still dilated to $7 \mathrm{~mm}$ in dark and only constricted slightly to $6 \mathrm{~mm}$ with light and near stimuli. Therefore, we ordered a magnetic resonance imaging study of the brain (with and without contrast) which did not reveal any abnormality.

Two months after the onset of anisocoria, the patient's pupil was reevaluated by a neuro-ophthalmologist. Although the left pupil was still $1 \mathrm{~mm}$ larger than the right one, the amount of anisocoria remained equal under dark or light conditions. There was no light-near dissociation. The left pupil did not show tonic constriction or redilation. One year later, the patient still had $1 \mathrm{~mm}$ anisocoria, which was equal in dim and bright light (right $5 \mathrm{~mm}$ and $4 \mathrm{~mm}$; left $6 \mathrm{~mm}$ and $5 \mathrm{~mm}$ ).

\section{Discussion}

We presented an unusual anisocoria case, with pharmacological mydriasis lasting more than 2 weeks that was caused by use of Similasan Pink Eye Relief OTC homeopathic eye drops. Although the patient's pupil started to respond to light and near stimuli one week after cessation of the drops, the anisocoria was not completely resolved one year after cessation of the drops.

There have been several previous reports of accidental pharmacological anisocoria associated with naphazoline (adrenergic agonist) in Opcon-A OTC eye drops, ${ }^{1}$ scopolamine patch via finger-eye contact, ${ }^{4}$ and ipratropium bromide (anticholinergic) nebulizer leakage. ${ }^{5}$ However, in these cases, the pupils returned to normal size in less than 3 days. The 2 -week period of pupil dilation in our patient might be explained by the nature of the belladonna ingredient in the homeopathic drop she used. The tropane alkaloids in belladonna consist of mainly atropine, hyoscyamine, and scopolamine. It has a higher concentration of hyoscyamine, ${ }^{6}$ which is the optical isomer of atropine. Hyoscyamine has stronger central and peripheral anticholinergic effects and is more potent in dilating the pupil. ${ }^{7,8}$ Therefore, it is possible that pupil dilation from hyoscyamine may last longer than dilation caused by atropine or scopolamine. 
Our patient stated that she noticed her left pupil was larger than her right one early in the morning; afterwards, she started the Similasan drops. It is possible that she could have had an unnoticed and undocumented physiological anisocoria. Although she had been examined yearly at our clinic for several years prior, her pupils had never been checked by the physician before dilation and were recorded as equal in size (with size various from 3.5 to $4.5 \mathrm{~mm}$ ) by the technicians who might not have noticed a small difference in pupil size. As a matter of fact, one year after the event, the physician documented the left pupil as $1 \mathrm{~mm}$ larger; however, the technician recorded equal pupil size upon her examination. There is also a remote possibility that the patient developed a new onset of Adie tonic pupil but failed to show supersensitivity to $0.1 \%$ pilocarpine because of the coexistence of anticholinergic mydriasis from the Similasan drops, and this was completely resolved 2 months later when she was reevaluated in the neuro-ophthalmology clinic. Thompson's study on over 100 Adie tonic pupil patients found the mean age of onset to be about 32.2 years, ranging from 5 years to 66 years (only one was older than 60 years), with recovery in accommodation during the first 2 years and loss of light response in more segments of iris sphincter. ${ }^{9}$ Our patient, however, would have been atypical for Adie tonic pupil, with an onset at age 70 and complete recovery within 2 months. The patient denied using any other drops, sprays, or patch. She had no risk for contact with tropane alkaloid plants while gardening since our agricultural zone 3 region is too harsh for these plants to grow.

It is challenging for ophthalmologists to understand the mechanisms and side effects of homeopathic OTC eye drops since most times there are no active chemical ingredients available, and the drug fact sheet lists only the names of the natural (botanical) source, which are most likely unfamiliar to most practitioners. Two of the nine types of Similasan eye drops, complete eye relief and irritated eye relief (previously called pink eye relief), contain belladonna, which can lead to pupil dilation, complicating the decision-making process during the work-up for anisocoria and the management of angle closure glaucoma. We believe it would be safer for patients/consumers if the manufacturers were required to, at the very least, list pupil dilation as a side effect and to include warnings such as "do not use if you have narrow angle glaucoma."

Anisocoria is an alarming clinical sign because of its close association with life-threatening conditions such as third nerve palsy from intracranial aneurysms, hemorrhage, neoplasms, or Horner's syndrome from carotid artery dissection, and pulmonary or brain tumors. Therefore, the clinical work-up for anisocoria often involves ordering highcost imaging studies. Our case of unilateral anticholinergic mydriasis caused by Similasan OTC drops highlights the importance, during the evaluation process, of understanding the pupil dilation effect from belladonna contained in some homeopathic eye drops.

\section{Acknowledgements}

The authors thank Marie Fleisner at Marshfield Clinic Research Institute for her assistance in manuscript preparation.

\section{References}

1. Williams TL, Williams AJ, Enzenauer RW. Case report: unilateral mydriasis from topical Opcon-A and soft contact lens. Aviat Space Environ Med. 1997;68:1035-1037.

2. Swayne JM. Survey of the use of homeopathic medicine in the UK health system. J R Coll Gen Pract. 1989;39:503-506.

3. Bulley FA. Observations on the use of Belladonna as a douche in some diseases of the eye. Prov Med J Retrosp Med Sci. 1842;4:3-5.

4. Ng J, Li Yim J. Accidental Unilateral Mydriasis from Hyoscine Patch in a Care Provider. Semin Ophthalmol. 2015;30:462463.

5. Santana-Cabrera L, Fernandez-Tagarro EJ, Del Amo-Nolasco B, Jaen-Sanchez N, Caceres-Agra JJ. Unilateral mydriasis secondary to ipratropium bromide in a critically ill patient. J Emerg Trauma Shock. 2012;5:199-200.

6. Hartmann T, Witte L, Oprach F, Toppel G. Reinvestigation of the Alkaloid Composition of Atropa belladonna Plants, Root Cultures, and Cell Suspension Cultures. Planta Med. 1986;(5):390-395.

7. Cushny AR. Atropine and the hyoscyamines-a study of the action of optical isomers. J Physiol. 1903;30:176-194.

8. Buckett WR, Haining CG. Some Pharmacological Studies on the Optically Active Isomers of Hyoscine and Hyoscyamine. Br J Pharmacol Chemother. 1965;24:138-146.

9. Thompson HS. Adie's syndrome: some new observations. Trans Am Ophthalmol Soc. 1977;75:587-626.

\section{Author Affiliations}

Lin Chen, MD, PhD*; Joseph C. Yeung, MD*; and Dennis R. Anderson, $M D \dagger$

*Department of Ophthalmology, Marshfield Clinic Minocqua Center, Minocqua, Wisconsin, USA $\dagger$ Department of Ophthalmology, Marshfield Clinic Marshfield Center, Marshfield, Wisconsin, USA 